Europhysics Letters

PREPRINT

\title{
Non-Ergodic Behaviour of the $k$-Body Embedded Gaussian Random Ensembles for Bosons
}

\author{
T. Asaga, L. Benet, T. Rupp and H. A. Weidenmüller \\ Max-Planck-Institut für Kernphysik, D-69029 Heidelberg, Germany
}

\begin{abstract}
PACS. 05.40.-a - Fluctuation phenomena, random processes, noise and Brownian motion. PACS. 05.30.Jp - Boson systems.

PACS. 21.10.-k - Properties of nuclei; nuclear energy levels.

PACS. 05.45.-a - Nonlinear dynamics and nonlinear dynamical systems.
\end{abstract}

\begin{abstract}
We investigate the shape of the spectrum and the spectral fluctuations of the $k$-body Embedded Gaussian Ensemble for Bosons in the dense limit, where the number of Bosons $m \rightarrow \infty$ while both $k$, the rank of the interaction, and $l$, the number of single-particle states, are kept fixed. We show that the relative fluctuations of the low spectral moments do not vanish in this limit, proving that the ensemble is non-ergodic. Numerical simulations yield spectra which display a strong tendency towards picket-fence type. The wave functions also deviate from canonical random-matrix behaviour.
\end{abstract}

Introduction. - Random-matrix theory (RMT) successfully describes the statistical behaviour of spectra and wave functions of a large variety of systems such as atoms, molecules, atomic nuclei and quantum dots [1, 2] . However, this RMT modeling is not completely realistic since all many-body systems are effectively governed by one- and two-body forces. This fact led to work on the two-body random ensembles for Fermions $|3| 6 \mid$ and to the introduction of the $k$-body embedded ensembles by Mon and French [7]. In the embedded ensembles, many-body states are constructed by distributing $m$ particles over $l$ degenerate single-particle levels. The matrix of the $k$-body interaction with $k \leq m$ is taken in this basis. For $k<m$, the $m$-body matrix elements of the random $k$-body interaction are correlated: The number of independent random variables is smaller than in RMT. Do these more realistic embedded ensembles yield the same results as RMT? Early numerical simulations for inter-

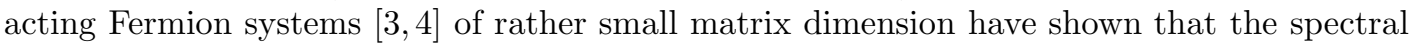
fluctuation properties of the embedded ensembles agree with those of RMT. Similar results were obtained in numerical simulations for Bosonic systems [8, 9 . Moreover, the Fermionic ensembles were shown to be ergodic. As far as we know, there are no results on the spectral ergodicity for Bosons.

Recently, three of the present authors introduced a novel analytical approach to the Fermionic embedded ensembles in the limit of infinite matrix dimension $(l \rightarrow \infty)$ [10]. The main results of this approach are: (i) For $2 k>m$, the average spectrum has semicircle shape, and the spectral fluctuation properties coincide with those of RMT; (ii) the spectral density changes shape at or near $2 k=m$ and becomes Gaussian; (iii) in the dilute limit $(k \ll m \ll l)$

(C) EDP Sciences 
the spectral fluctuations are completely uncorrelated (Poissonian); (iv) the spectral fluctuations change gradually from Wigner-Dyson to Poisson.

In this Letter, we extended our work to the case of Bosons. As in the Fermionic case, we consider the limit of infinite matrix dimension. For Bosons this limit is realized either by letting $l \rightarrow \infty$ (the same limit as for Fermions), or by letting $m \rightarrow \infty$ with $k$ and $l$ fixed. This second case, the dense limit, is novel and has no analogue in the Fermionic case. In the following, we focus attention exclusively on the dense limit. We prove analytically that in this limit, the ensemble is not ergodic. Numerical results for the spectral correlations are obtained by both ensemble unfolding and by spectral unfolding. In the latter case we find a strong tendency of the spectra towards picket-fence behaviour. We also show that some eigenfunctions display Fock-space localization. Details of the derivations and a complete treatment including the limit $l \rightarrow \infty$, are given in Ref. [11.

Definitions. - We consider $m$ spinless Bosons in $l$ degenerate single-particle states with associated creation and annihilation operators $b_{j}^{\dagger}$ and $b_{j}$ where $j=1, \ldots, l$. Hilbert space is spanned by the $N=\left(\begin{array}{c}l+m-1 \\ m\end{array}\right)$ orthonormal $m$-particle states $|\mu\rangle(\mu=1, \ldots, N)$, written as $|\mu\rangle=\left[\mathcal{N}\left(j_{1}, \ldots, j_{m}\right)\right]^{-1} b_{j_{1}}^{\dagger} \ldots b_{j_{m}}^{\dagger}$ with $j_{1} \leq j_{2} \leq \ldots \leq j_{m}$ and $\mathcal{N}\left(j_{1}, \ldots, j_{m}\right)$ a normalization constant. The states $|\mu\rangle$ are equivalently characterized by a sequence of occupation numbers $\left(n_{1}, \ldots, n_{l}\right)$ of the $l$ single-particle states with $b_{j_{1}}^{\dagger} \ldots b_{j_{m}}^{\dagger}=\left(b_{1}^{\dagger}\right)^{n_{1}} \ldots\left(b_{l}^{\dagger}\right)^{n_{l}}$. Setting $\mathcal{N}\left(j_{1}, \ldots, j_{m}\right)=\sqrt{n_{1} ! \ldots n_{l} !}$ normalizes all $m$-particle states $|\mu\rangle$ to 1 .

The bosonic $m$-particle states are coupled through a random $k$-body interaction $V_{k}(\beta)$ with $k \leq m$, given by

$$
V_{k}(\beta)=\sum_{\substack{1 \leq j_{1} \leq j_{2} \leq \ldots \leq j_{k} \leq l \\ 1 \leq i_{1} \leq i_{2} \leq \ldots \leq i_{k} \leq l}} v_{j_{1}, \ldots, j_{k} ; i_{1}, \ldots, i_{k}} \frac{b_{j_{1}}^{\dagger} \ldots b_{j_{k}}^{\dagger} b_{i_{k}} \ldots b_{i_{1}}}{\mathcal{N}\left(j_{1} \ldots j_{k}\right) \mathcal{N}\left(i_{1} \ldots i_{k}\right)}
$$

We refer to $k$ as to the rank of the interaction. As in the canonical case, the labels $\beta=1$ and $\beta=2$ denote the orthogonal and the unitary ensemble, respectively. The matrix element $v_{j_{1}, \ldots, j_{k} ; i_{1}, \ldots, i_{k}}$ of the $k$-body interaction taken between the single-particle states $j_{1}, \ldots, j_{k}$ and $i_{1}, \ldots, i_{k}$ is totally symmetric with respect to $j_{1}, \ldots, j_{k}$ and $i_{1}, \ldots, i_{k}$. The elements differing in the sequence of indices $\left\{j_{1} \ldots j_{k} ; i_{1} \ldots i_{k}\right\}$ (except for permutations of $\left\{j_{1} \ldots j_{k}\right\}$ and of $\left\{i_{1} \ldots i_{k}\right\}$ and for symmetries specified by $\beta$ ) are uncorrelated Gaussian-distributed random variables with zero mean and a common second moment $v_{0}^{2}$. Without loss of generality we put $v_{0}^{2}=1$ in the sequel. The normalization coefficients $\mathcal{N}\left(j_{1}, \ldots, j_{k}\right)$ and $\mathcal{N}\left(i_{1}, \ldots, i_{k}\right)$ in Eq. (11) guarantee that for $k=m$, the embedded ensembles are identical to the canonical ensembles of random-matrix theory. This defines the Bosonic $k$-body embedded Gaussian orthogonal (unitary) ensemble of random matrices, respectively, in short BEGOE $(k)$ and $\operatorname{BEGUE}(k)$. The number of independent random variables is given by $K_{\beta}=\beta\left(\begin{array}{c}l+k-1 \\ k\end{array}\right)\left[\left(\begin{array}{c}l+k-1 \\ k\end{array}\right)+\delta_{\beta 1}\right] / 2$.

The Second Moment. - By virtue of the randomness of $V_{k}(\beta)$, the elements of the matrix $\left\langle\nu\left|V_{k}(\beta)\right| \mu\right\rangle$ are random variables with a Gaussian probability distribution and zero mean value. The spectral properties are completely determined by the second moment

$$
\begin{aligned}
B_{\mu \nu, \rho \sigma}^{(k)}(\beta) & =\overline{\left\langle\mu\left|V_{k}(\beta)\right| \sigma\right\rangle\left\langle\rho\left|V_{k}(\beta)\right| \nu\right\rangle} \\
& =\sum_{\alpha(k), \gamma(k)}\left\langle\mu\left|\mathcal{B}_{\alpha(k)}^{\dagger} \mathcal{B}_{\gamma(k)}\right| \sigma\right\rangle\left[\left\langle\rho\left|\mathcal{B}_{\gamma(k)}^{\dagger} \mathcal{B}_{\alpha(k)}\right| \nu\right\rangle+\delta_{\beta 1}\left\langle\nu\left|\mathcal{B}_{\gamma(k)}^{\dagger} \mathcal{B}_{\alpha(k)}\right| \rho\right\rangle\right] .
\end{aligned}
$$

The overbar denotes the average over the ensemble. We have simplified the notation by introducing the operators $\mathcal{B}_{\alpha(r)}^{\dagger}=\left[\mathcal{N}\left(j_{1}, \ldots, j_{r}\right)\right]^{-1} b_{j_{1}}^{\dagger} \ldots b_{j_{r}}^{\dagger}$ and the adjoints $\mathcal{B}_{\alpha(r)}$. The 
index $\alpha(r)$ is a short-hand notation for the rank $r$ and for the sequence of indices $\left\{j_{1} \ldots j_{r}\right\}$. The matrix $B^{(k)}(\beta)$ is Hermitean in the pairs of indices $(\mu, \nu)$ and $(\rho, \sigma)$. It is easy to prove the "duality" relation $B_{\mu \nu, \rho \sigma}^{(k)}(2)=B_{\mu \sigma, \rho \nu}^{(m-k)}(2)$ which for $\beta=2$ connects the second moments of the $k$-body and the $(m-k)$-body interactions. For $k=m$ and $\beta=2$, the right-hand side of Eq. (2) reduces to $\delta_{\mu \nu} \delta_{\rho \sigma}$, and correspondingly for $\beta=1$. This shows that for $k=m$, $\operatorname{BEGUE}(k)$ and $\operatorname{BEGOE}(k)$ reduce to the GUE and GOE, respectively.

In the Fermionic case, results for the shape of the spectrum and for the spectral fluctuations were obtained with the help of the eigenvector decomposition of the second moment. A similar decomposition exists in the case of Bosons and is derived in Ref. [11. In the following, we only state the results of this derivation. We solve the eigenvalue equation $\sum_{\rho \sigma} B_{\mu \nu, \rho \sigma}^{(k)}(2) C_{\sigma \rho}^{(s a)}=$ $\Lambda^{(s)}(k) C_{\mu \nu}^{(s a)}$, with eigenvectors $C^{(s a)}$ and with eigenvalues $\Lambda^{(s)}(k)$ given by

$$
\Lambda^{(s)}(k)=\left(\begin{array}{c}
m-s \\
k
\end{array}\right)\left(\begin{array}{c}
l+m+s-1 \\
k
\end{array}\right) \text {. }
$$

Here, $s=0, \ldots, m$ while $a$ labels the degenerate eigenvectors. For the degree of degeneracy $D^{(s)}$ of the eigenvalues $\Lambda^{(s)}(k)$ we find $D^{(0)}=1$ and $D^{(s)}=\left(\begin{array}{c}l+s-1 \\ s\end{array}\right)^{2}-\left(\begin{array}{c}l+s-2 \\ s-1\end{array}\right)^{2}$ for $s \geq 1$. It follows that $\sum_{s=0}^{m} D^{(s)}=N^{2}$ showing that the eigenvectors form a complete set. We choose Hermitean linear combinations of the degenerate eigenvectors which obey the orthonormality condition $\sum_{\mu \nu} C_{\mu \nu}^{(s a)} C_{\nu \mu}^{(t b)}=N \delta_{s t} \delta_{a b}$. These results can be extended to $\beta=1$. Hence, the matrix $B^{(k)}(\beta)$ can be expanded as

$$
B_{\mu \nu, \rho \sigma}^{(k)}(\beta)=\frac{1}{N} \sum_{s=0}^{m} \Lambda^{(s)}(k) \sum_{a}\left[C_{\mu \nu}^{(s a)} C_{\rho \sigma}^{(s a)}+\delta_{\beta 1} C_{\mu \rho}^{(s a)} C_{\nu \sigma}^{(s a)}\right] .
$$

Low Moments of $V_{k}$. - Using the eigenvector decomposition of $B^{(k)}(\beta)$, duality and the orthonormality of the $C^{(s a)}$ 's, explicit expressions for the low moments of $V_{k}(\beta)$ are obtained. From these we calculate three ratios that yield information on the shape of the spectral density. The ratio $S$ measures the fluctuations of the center of the spectrum in units of the average width of the spectrum. The ratio $R$ measures the relative fluctuations of the width of the spectrum. The ratio $Q$ is related to the kurtosis $\kappa=Q+2$ and marks the difference between the semicircular $(Q=0)$ and the Gaussian shape $(Q=1)$. The definitions of $S, R$, and $Q$ are given in Ref. [10].

We are particularly interested in the behaviour of the three ratios in the dense limit, i.e. the case $N \rightarrow \infty$ attained by letting $m \rightarrow \infty$ while keeping both $l$ and $k$ fixed. We obtain

$$
\begin{aligned}
\lim _{m \rightarrow \infty} S(k, m, l) & =\frac{\left(1+\delta_{\beta 1}\right)\left(\begin{array}{c}
2 k \\
k
\end{array}\right)\left(\begin{array}{c}
l+k-1 \\
k
\end{array}\right)^{-1}}{\left(\begin{array}{c}
2 k \\
k
\end{array}\right)+\delta_{\beta 1} \sum_{s=0}^{k}\left(\begin{array}{c}
2 k \\
k+s
\end{array}\right)\left(\begin{array}{c}
l+k+s-1 \\
k+s
\end{array}\right)^{-1} d^{(s)}}, \\
\lim _{m \rightarrow \infty} R(k, m, l) & =\frac{2 \sum_{s=0}^{k}\left[\left(\begin{array}{c}
2 k \\
k+s
\end{array}\right)\left(\begin{array}{c}
l+k+s-1 \\
k+s
\end{array}\right)^{-1}\right]^{2}\left[D^{(s)}+\delta_{\beta 1}\left(D^{(s)}+2 d^{(s)}\right)\right]}{\left[\left(\begin{array}{c}
2 k \\
k
\end{array}\right)+\delta_{\beta 1} \sum_{s=0}^{k}\left(\begin{array}{c}
2 k \\
k+s
\end{array}\right)\left(\begin{array}{c}
l+k+s-1 \\
k+s
\end{array}\right)^{-1} d^{(s)}\right]^{2}}, \\
\lim _{m \rightarrow \infty} Q(k, m, l) & =\sum_{s=0}^{k}\left(\begin{array}{c}
2 k \\
k
\end{array}\right)^{-1}\left(\begin{array}{c}
2 k \\
k+s
\end{array}\right)\left(\begin{array}{c}
l+k+s-1 \\
k+s
\end{array}\right)^{-1} D^{(s)}=1,
\end{aligned}
$$

with $d^{(s)}=\left(\begin{array}{c}l+s-2 \\ s\end{array}\right)$. Eq. (7) applies only in the unitary case $(\beta=2)$ and implies that in the dense limit, the average spectrum has Gaussian shape. We have not been able to extend this 


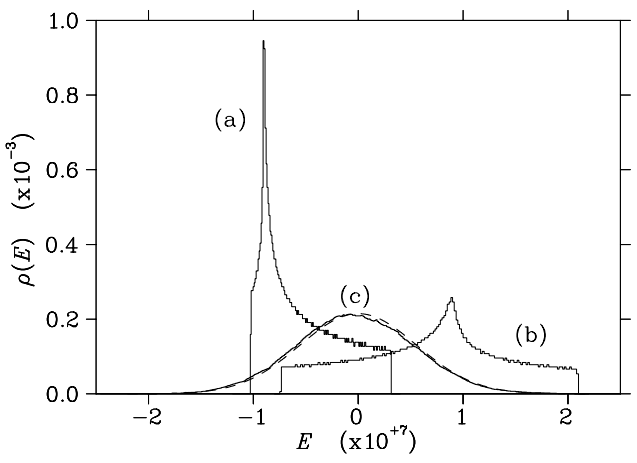

Fig. 1

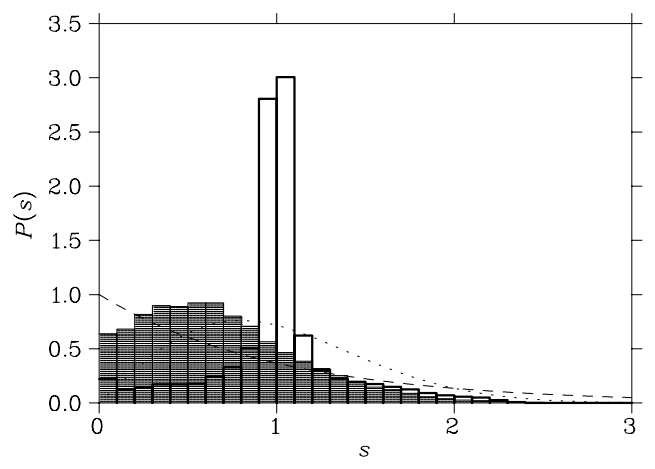

Fig. 2

Fig. 1 - Spectral density of the BEGOE(2) for $l=2$ and $m=3000$, normalized to the dimension $N=3001$ of Hilbert space. The curves (a) and (b) show the level densities of two members of the ensemble; curve (c) shows the ensemble-averaged spectral density. The dashed curve shows the theoretically predicted Gaussian shape of the average spectrum.

Fig. 2 - Nearest-neighbour spacing distribution $P(s)$ obtained by ensemble unfolding (filled histogram) and spectral unfolding (empty histogram). The dotted curve gives the Wigner surmise and the dashed one the Poisson distribution.

analytical result to the orthogonal case $\beta=1$. On physical grounds, however, an equation analogous to Eq. (7) is expected to be valid also for the $\operatorname{BEGOE}(k)$. We conclude that the shape of the average spectrum has Gaussian form. This is in keeping with the results of Ref. [13].

A more important and surprising result lies in the fact that the right-hand sides of Eqs. (5) and (6) do not vanish: The fluctuations of the centroids and of the variances of individual spectra do not vanish asymptotically. This feature differs from the behaviour both of canonical RMT and of the embedded Fermionic ensembles. We are led to the important conclusion that the Bosonic ensembles are not ergodic in the dense limit $m \rightarrow \infty$ with $k$ and $l$ fixed. Nonergodicity appears to be a consequence of the fact that the number of independent random variables $K_{\beta}$ in the ensemble does not grow with $m$, but stays finite for fixed $k$ and $l$.

Numerical results. - For lack of analytical techniques, we use numerical simulations to obtain infromation on the spectral fluctuation properties of $\operatorname{BEGOE}(k)$ in the dense limit. With $l=2$ and $k=2$, the dimension of Hilbert space is given by $N=m+1$, and $m \gg l$ is easily attainable numerically. The number of independent random variables is $K_{1}=6$. The many-particle states $\left|\mu_{n}\right\rangle$ are written as $(m-n, n)$ where $m-n$ and $n$ indicate the number of Bosons occupying the first and the second single-particle state, respectively. In the basis $\left\{\left|\mu_{0}\right\rangle,\left|\mu_{1}\right\rangle, \ldots,\left|\mu_{m}\right\rangle\right\}$, the Hamiltonian matrix attains band structure, with non-zero matrix elements on the main diagonal and on the $k$ closest diagonals.

Fig. 1 1 shows the level densities of two members of the ensemble and the ensemble-averaged density obtained from 1512 spectra $(m=3000)$. We have also plotted a Gaussian density whose width is given by the theoretical prediction [11] and which agrees with the shape of the ensemble-averaged density. The striking differences between each of the two spectral densities and between these and the ensemble-averaged spectral density illustrate the non-ergodic character of $\operatorname{BEGOE}(2)$ for $m \gg l$. The Gaussian form of the ensemble-averaged spectrum arises as a consequence of the Central Limit Theorem. It has no bearing on individual spectral shapes. 


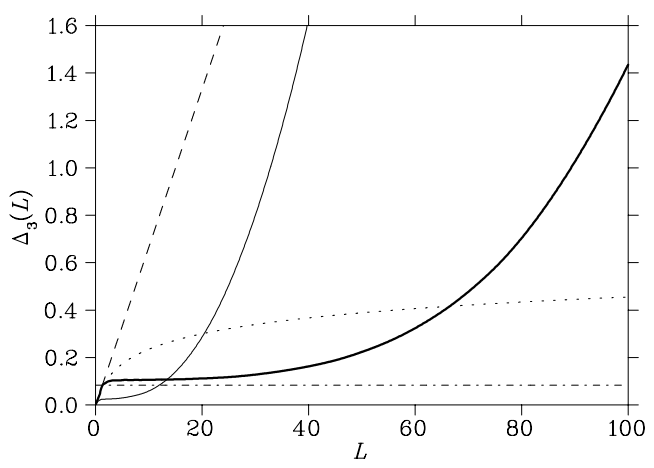

Fig. 3

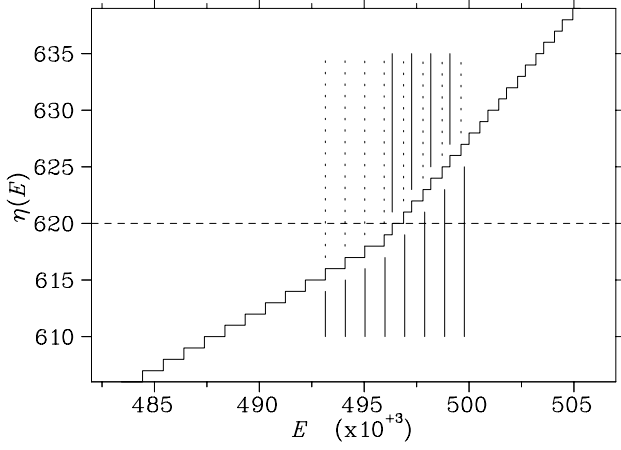

Fig. 4

Fig. $3-\Delta_{3}$-statistics measured at the centers of the spectra after ensemble unfolding (thin solid line) and spectral unfolding (bold line). For comparison we have plotted the results for the GOE (dotted curve), for a Poissonian spectrum (dashed line), and for a picket-fence spectrum (dotted-dashed line).

Fig. 4 - Detail of the staircase function $\eta(E)$ for a typical member of BEGOE(2) with $m=1000$ and $l=2$. The vertical lines help to show how two (almost) equidistant spectra overlap around the level 620. We note the change in the density of states after this level.

The evaluation of measures of spectral fluctuations requires the spectra to be unfolded. Unfolding can be done either individually for each spectrum (spectral unfolding) or by a single transformation which is applied to all spectra of the ensemble (ensemble unfolding). We employed both types of unfolding in our statistical analysis. For spectral unfolding, a polynomial was fitted to the staircase function of each realization of a spectrum. Starting from the value unity, we changed the degree of the polynomial for each realization until the first minimum of the associated $\chi^{2}$ distribution was reached. The maximum degree considered was 20 . The first minimum was typically found around degree 11. Ensemble unfolding was carried out by first averaging the staircase functions over the ensemble. The averaged staircase function was fitted by a polynomial of degree 11. We have analyzed 1512 realizations for $m=3000$. After fitting the polynomials by including all levels of each spectrum for both spectral and ensemble unfolding, we only considered $60 \%$ of all levels, i.e., 1800 levels for each realization located in the centre part of each spectrum.

In Figs. 2 and 3 we show the nearest-neighbour spacing distribution $P(s)$ and the $\Delta_{3}-$ statistics obtained for $m=3000$ by ensemble unfolding and by spectral unfolding. In both cases the distribution $P(s)$ corresponds neither to the Wigner surmise nor to a Poisson distribution. In the case of ensemble unfolding the level repulsion characteristic of the GOE is clearly lost. For spectral unfolding the distribution $P(s)$ is dominated by a prominent peak centered at $s=1$ suggesting that individual spectra have an almost constant level spacing. The $\Delta_{3}$-statistics obtained by ensemble unfolding deviates rapidly from GOE behaviour and increases almost linearly. In the case of spectral unfolding, $\Delta_{3}(L)$ is almost constant up to $L \sim 20$. This result again suggests a tendency of individual spectra towards a picket-fence-like behaviour. Beyond this range, $\Delta_{3}(L)$ grows albeit less rapidly than for ensemble unfolding.

We turn to the detailed structure of individual spectra. Fig. A shows the staircase function $\eta(E)$ for a typical member of the ensemble $(k=2, l=2, m=1000)$. The spectrum is dominated by levels with almost constant spacing. The staircase function typically displays one or more points where an abrupt change in the density of states takes place. In Fig. 团, the spectrum has (almost) constant spacing up to the level 619. From level 620 on, the spacing 

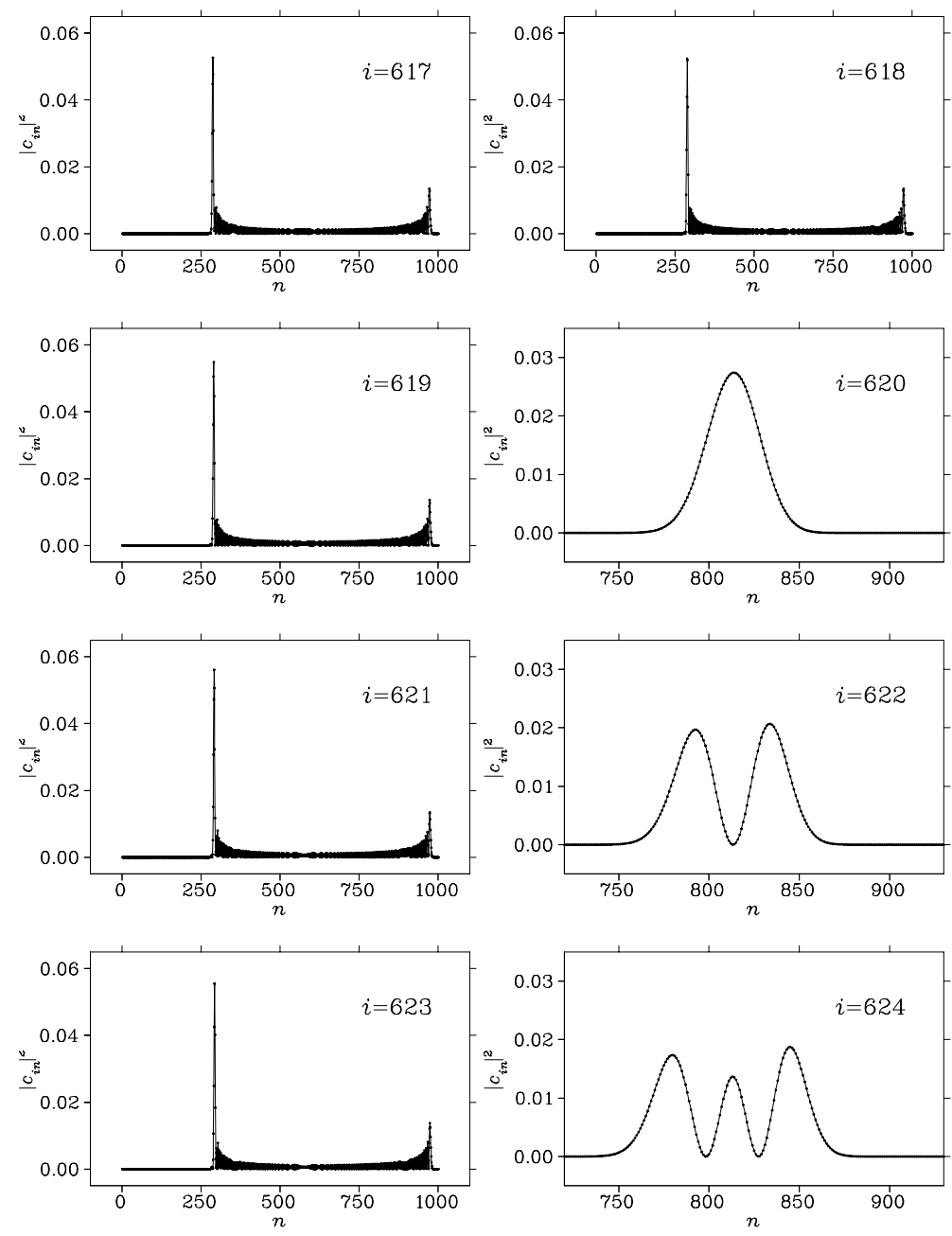

Fig. 5 - Probabilities $\left|c_{i n}\right|^{2}$ for eigenvectors belonging to eigenvalues in the vicinity of the kink shown in Fig. 4. The two overlapping segments of nearly equidistant levels are easily distinguished by the structure of the eigenfunctions.

between neighbouring levels is no longer constant. However, the spacing between next-tonearest-neighbours does have this property and is almost the same as the nearest-neighbour spacing before level 620 . This is illustrated by the vertical lines plotted above and below the staircase function. These observations imply non-stationary properties of the spectra. In addition, our result suggests that the spectrum of an individual member of the ensemble consists of pieces of overlapping segments of spectra with almost constant level spacings. The kink at level 620 in Fig. 4 marks the left edge of the overlap region.

The overlap of two segments of almost equidistant levels also influences the structure of the eigenfunctions. We write the eigenvectors $|i\rangle$ as linear combinations of the ordered many-body basis states $\left|\mu_{n}\right\rangle,|i\rangle=\sum_{n=0}^{m} c_{i n}\left|\mu_{n}\right\rangle$. In Fig. 司 we plot the probabilities $\left|c_{i n}\right|^{2}$ of eigenvectors $|i\rangle$ belonging to eigenvalues in the vicinity of the kink shown in Fig. A. Eigenvectors up to $i=619$ behave similarly and are somewhat extended, although the distribution of the 
intensities clearly deviates from the Porter-Thomas distribution expected from RMT. This behaviour changes abruptly at $i=620$. We emphasize the difference in the scales used in Fig. 5 for $i=619$ and for the even $i$ values starting with $i=620$. The eigenvectors with $i=621,623$ display the same behaviour as the eigenvectors up to $i=619$ and, thus, correspond to the first segment of the two equidistant spectra. The eigenvectors with $i=620,622,624$ are much more localized in Fock space. They differ in the number of intensity oscillations. As $i$ increases, so does this number, and the levels on this second segment become more and more delocalized. At some point the spread of the eigenfunctions in the second segment is indistinguishable from that in the first one.

Conclusions. - We have studied the spectral behaviour of the Bosonic embedded ensembles in the dense limit attained by letting $m \rightarrow \infty$ with $l$ and $k$ fixed. We have shown that in this limit, both the $\operatorname{BEGOE}(k)$ and the $\operatorname{BEGUE}(k)$ are non-ergodic. To the best of our knowledge, this is the first case of a random-matrix model for which such non-ergodic behaviour has been established in the limit of infinite matrix dimension. Moreover, we have shown that the spectral fluctuations deviate strongly from RMT results. This result disagrees with the conclusions based on numerical simulations of Refs. [8, 9]. We ascribe this disagreement to the fact that the ratios $m / l$ considered in Refs. [8, 9] were too small to produce deviations from Wigner-Dyson statistics. Our numerical simulations provide evidence for both a nonstationary and a picket-fence-type of behaviour in both short- and long-range correlations of the spectra. More precisely, individual spectra seem generically to consist of overlapping segments of spectra of picket-fence type. In the overlap region, the eigenfunctions of the levels in the two segments differ markedly, the eigenfunctions in the second segment displaying strong localization in Fock space. We have not yet attained an analytical understanding of these properties which we attribute to the small number of independent random variables $K_{\beta}$ (with $K_{\beta}$ independent of $m$ ), and to the specific structure of the Hamiltonian matrix of the Bosonic ensembles. In particular, we do not know how the spectra change as $l$ increases.

We are grateful to O. Bohigas and T.H. Seligman for stimulating discussions and useful suggestions. T.A. acknowledges support from the Japan Society for the Promotion of Science.

\section{REFERENCES}

[1] Brody T.A. et al., Rev. Mod. Phys., 53 (1981) 385.

[2] Guhr T., Müller-Groeling A., and Weidenmüller H.A., Phys. Rep., 299 (1998) 189.

[3] French J.B. and Wong S.S.M., Phys. Lett. B, 35 (1971) 5.

[4] Bohigas O. and Flores J., Phys. Lett. B, 34 (1971) 261; Phys. Lett. B, 35 (1971) 383.

[5] Gervois A., Nucl. Phys., A 184 (1972) 507.

[6] French J.B., Revista Mexicana de Fisica, 22 (1973) 221.

[7] Mon K.K. and French J.B., Ann. Phys. (N.Y.), 95 (1975) 90.

[8] Manfredi V.R., Lett. Nuovo Cimento, 40 (1984) 135.

[9] Patel K., Desai M.S., Potbhare V. and Kota V.K.B., Phys. Lett. A, 275 (2000) 329.

[10] Benet L., Rupp T. and Weidenmüller H.A., Phys. Rev. Lett., 87 (2001) 010601; Ann. Phys. (N.Y.), (2001) in press (cond-mat/0010426).

[11] Asaga T., Benet L., Rupp T. and Weidenmüller H.A.,, cond-mat/0107364, submitted.

[12] Flores J., Horoi M., Müller M. and Seligman T.H., Phys. Rev. E, 63 (2001) 026204.

[13] Kota V.K.B. and Potbhare V., Phys. Rev. C, 21 (1980) 2637. 